\title{
Prescrições de medicamentos sujeitos a controle especial em um município do Seridó Potiguar, Brasil
}

\author{
Drug subject to special control prescription in a County of Seridó \\ Potiguar, Brazil
}

Recebido em: $12 / 12 / 2020$ Aceito em: 20/04/2021
Lucas Soares da Nóbrega SILVA; Maria Vitória Ideão Leite da ROCHA; Almária Mariz BATISTA Escola Multicampi de Ciências Médicas do Rio Grande do Norte, Universidade Federal do Rio Grande do Norte. Av. Dr. Carlindo Dantas, 540, $2^{\circ}$ andar, CEP 59300-000. Caicó, RN, Brasil. E-mail:almariamariz@yahoo.com.br

\section{ABSTRACT}

The prescription of medications constitutes a therapeutic intervention that must consider technical-legal and user-centered aspects to achieve effective therapy and patient safety. Thus, this study aimed to evaluate prescriptions of the subject to special control drugs in primary care in Caicó, RN, Brazil, based on the legislation in force, through a cross-sectional, descriptive-exploratory, quantitative study, based on the special control prescription prepared by physicians during the period from July 2018 to June 2019, at the municipal Pharmaceutical Supply Center. Pharmacological classification of prescription drugs was also analyzed and their compliance with the Municipal List of Essential Medicines. In all, 1.682 prescriptions were evaluated; $1.7 \%$ of these were eliminated due to readability issues, meaning they already were legislation non-compliant. There was a predominance of non-compliant prescriptions (99.77\%), whose items duration of treatment (97.1\%), route of administration (89.3\%), and drug nomenclature by active ingredient $(14.6 \%)$ were the most prevalent regarding these non-conformities. As for the pharmacological classes of the prescribed drugs, benzodiazepine anxiolytics predominated (30.97\%). All prescription drugs complied with the municipal essential drug list. There is a need to reorient medical professional training, through insertion and/or emphasis on strategies for preparing prescriptions in compliance with technical-legal and user-centered precepts, in addition to interprofessional education and work, in order to contribute to user-centered assistance, improved patient safety, access, and effectiveness in the context of health care.

Keywords: drug prescription; psychopharmaceuticals; primary health care.

\section{RESUMO}

A prescrição de medicamentos constitui uma intervenção terapêutica e deve considerar aspectos técnico-legais e usuário-centrados para alcançar a efetividade da terapia e a segurança do paciente. Desta forma, foram avaliadas prescrições de medicamentos sujeitos a controle especial na atenção primária do município de Caicó, RN, com base na legislação em vigor, por meio de um estudo transversal, 
descritivo-exploratório, quantitativo, baseado no receituário de controle especial elaborado por médicos durante o período de julho de 2018 a junho de 2019, arquivado na Central de Abastecimento Farmacêutico municipal. Também foi analisada a classificação farmacológica dos medicamentos prescritos, bem como sua conformidade com a Relação Municipal de Medicamentos Essenciais. Ao todo, foram avaliadas 1.682 prescrições. Houve predomínio de prescrições não conformes $(99,77 \%)$, cujos itens duração do tratamento $(97,1 \%)$, via de administração $(89,3 \%)$ e nomenclatura do medicamento por princípio ativo $(14,6 \%)$ foram os mais prevalentes quanto a não conformidades. Quanto às classes farmacológicas dos medicamentos prescritos, predominaram ansiolíticos benzodiazepínicos $(30,97 \%)$. Todos os medicamentos prescritos estavam em conformidade com a lista de medicamentos essenciais municipal. Depreende-se a necessidade de reorientação da formação profissional médica, através de inserção e/ou maior ênfase a estratégias para elaboração de prescrições em observância a preceitos técnico-legais e usuário-centrados, além de educação e trabalho interprofissionais, a fim de contribuir para assistência usuário-centrada, melhoria de segurança do paciente, acesso e efetividade no contexto da assistência à saúde.

Palavras-chave: prescrição; psicofármacos; atenção primária à saúde.

\section{INTRODUÇÃO}

A prescrição medicamentosa é uma ordem escrita de um plano terapêutico elaborado por profissional legalmente habilitado, dirigida ao farmacêutico, definindo como o fármaco deve ser fornecido ao paciente, e a este, determinando as condições em que o fármaco deve ser utilizado. Entretanto, tais instruções podem ser inefetivas ou ter a segurança terapêutica comprometida, caso o paciente ou seus familiares não entenderem o que foi prescrito $(1,2)$.

O custo associado à ocorrência de eventos adversos a medicamentos foi estimado, globalmente, em US\$ 42 bilhões/ano. Por outro lado, a literatura disponível no Brasil sobre erros de medicação ainda é insuficiente. Há mais publicações sobre reações adversas a medicamentos do que sobre erros de medicação $(3,4)$.

Outrossim, uma revisão sistemática sobre incidentes de segurança na atenção primária mostrou incidentes relacionados a diagnóstico e medicação, com os erros de prescrição como os mais prevalentes (5).

Diante dessa conjuntura, discussões acerca de práticas seguras relacionadas a medicamentos têm se destacado. Quanto à prescrição, por exemplo, deve conter, de forma objetiva, legível e dentro dos padrões definidos pelos órgãos reguladores, todas as orientações sobre o tratamento medicamentoso e/ou não medicamentoso a ser seguido pelo paciente (2). Isto têm resultado em iniciativas globais e locais visando à promoção do uso racional de medicamentos.

A Organização Mundial de Saúde (OMS) propôs indicadores de qualidade de prescrição na atenção primária (6) e lançou, em 2017, o Terceiro Desafio Global de Segurança do Paciente (Medicação Sem Danos) cuja meta foi a redução de erros de medicação em 50\% no período de 2018 a 2022 (7).

No Brasil, foi instituído o Programa Nacional de Segurança do Paciente (8) que, entre outros, fomentou os Protocolos Básicos de Segurança do Paciente, com destaque ao Protocolo de Segurança $\mathrm{Na}$ Prescrição, Uso e Administração de Medicamentos (9). Outrossim, a prescrição de medicamentos é regulamentada por normas como Lei $\mathrm{n}^{\circ} 5.991 / 73$ (10), Portaria ${ }^{\circ} 344 / 98$ (11), Lei no $9.787 / 99$ (12) e RDC n ${ }^{\circ} 20 / 11$ (13).

Dentre os medicamentos sujeitos a controle especial, regidos pela Portaria $n^{\circ} 344 / 98$, estão os entorpecentes e psicotrópicos, os quais podem determinar dependência física ou psíquica e compõem as listas A1, A2, A3, B1 e B2 desta Portaria; alguns são usados para tratamento de problemas mentais e de comportamento, assim como alguns medicamentos da lista $\mathrm{C} 1$ (11). 
Nessa conjuntura, estima-se que uma em cada dez pessoas precise de cuidados de saúde mental em qualquer momento e cada US\$ 1 investido na ampliação do tratamento para doenças mentais comuns, como depressão e ansiedade, leva a um retorno de US\$ 4 em melhor saúde e capacidade de trabalho (14).

Diante desse contexto, foram avaliadas prescrições de medicamentos sujeitos a controle especial no âmbito da atenção primária à saúde do município de Caicó, RN, Brasil, com base na legislação em vigor.

\section{MÉTODO}

Foi desenvolvido um estudo transversal, de caráter descritivo-exploratório, com abordagem quantitativa, na Central de Abastecimento Farmacêutico (CAF) de Caicó, RN, localizado na região Seridó Potiguar, a qual também abrange os municípios Acari, Bodó, Carnaúba dos Dantas, Cerro Corá, Cruzeta, Currais Novos, Equador, Florânia, Ipueira, Jardim de Piranhas, Jardim do Seridó, Jucurutu, Lagoa Nova, Ouro Branco, Parelhas, Santana do Matos, Santana do Seridó, São Fernando, São João do Sabugi, São José do Seridó, São Vicente, Serra Negra do Norte, Tenente Laurentino Cruz e Timbaúba dos Batistas (15).

O objeto desta pesquisa foi o receituário de controle especial elaborado durante o período de julho de 2018 a junho de 2019, arquivado na CAF.

Foram incluídas, nesse estudo, prescrições de médicos lotados nas unidades básicas de saúde (UBS). Prescrições provenientes de outros serviços de saúde, prescrições de cirurgiões-dentistas, transcrições de Enfermagem bem como prescrições ilegíveis não foram incluídas.

Com base na Lei ${ }^{0}$ 5.991/73 (10), as prescrições foram avaliadas quanto à presença de nome do paciente, carimbo, assinatura e categoria profissional do prescritor, data da prescrição, legibilidade, via de administração, dosagem/concentração e duração do tratamento dos medicamentos prescritos.

Considerando a Portaria $n^{\circ} 344 / 98$ (11), foram analisadas correspondência entre categorização do medicamento e respectivo tipo de prescrição, correspondência entre quantidade de medicamento e respectivo tipo de prescrição, correspondência entre unidade posológica e respectivo tipo de prescrição e identificação do receptor.

A nomenclatura dos medicamentos prescritos por princípio ativo foi utilizada como item de avaliação em consonância com a Lei no 9.787/99 (12).

Para cada item avaliado, com exceção da classe farmacológica, havia duas respostas possíveis, sendo que para os itens nome do paciente, carimbo e assinatura do prescritor, data da prescrição, via de administração, concentração e duração do tratamento, as duas respostas possíveis foram conforme ou não conforme. Prescrições que não apresentavam, concomitantemente, carimbo e assinatura, foram consideradas não conformes.

A classificação farmacológica foi baseada no sistema Anatomical Therapeutic Chemical (ATC) (16). Além disso, foi verificado se os medicamentos prescritos constavam na Relação Municipal de Medicamentos Essenciais (REMUME 2014) (17).

A coleta dos dados foi realizada mediante aprovação pelo Comitê de Ética do Hospital Universitário Onofre Lopes (CEP/HUOL), sob $n^{\circ}$ de protocolo 64367517.3.0000.5292

Os dados foram tabulados e analisados via software Microsoft Excel, por meio de estatística descritiva, baseada em gráficos, tabelas e frequências absoluta e relativa.

\section{RESULTADOS E DISCUSSÃO}

Ao todo, foram avaliadas 1.682 prescrições (Tabela 1) das quais apenas 4 (0,24\%) estavam conformes com a legislação pertinente quanto aos itens avaliados conforme a Figura 1.

Quanto à prevalência de itens não conformes com a legislação, houve predomínio de itens relacionados a posologia do medicamento, no caso, duração do tratamento $(97,1 \%)$ e via de administração (89,3\%), o que corrobora um estudo de avaliação de receituário de controle especial em UBS de Jequié, BA, em que 95,7\% não constava a duração do tratamento (18). Isto impacta no processo de dispensação de medicamentos pelo 
Tabela 1. Distribuição dos itens não conformes com a legislação, em receitas emitidas no período de julho de2018 a junho de 2019, arquivadas na Central de Abastecimento Farmacêutico (CAF) de Caicó, RN, Brasil.

\begin{tabular}{|c|c|c|}
\hline \multirow{2}{*}{ Item } & \multicolumn{2}{|c|}{ Prescrições não conformes $(\mathrm{N}=1.682)$} \\
\hline & FA & FR $(\%)$ \\
\hline \multicolumn{3}{|l|}{ Lei n $5.991 / 73$} \\
\hline Informações que permitem contatar prescritor & 0 & 0 \\
\hline Duração de tratamento & 1.661 & 97,1 \\
\hline Carimbo e assinatura do prescritor & 0 & 0 \\
\hline Data & 0 & 0 \\
\hline Concentração & 0 & 0 \\
\hline Via de administração & 1.528 & 89,3 \\
\hline Nome do medicamento & 0 & 0 \\
\hline Identificação do usuário & 0 & 0 \\
\hline \multicolumn{3}{|l|}{ Lei n 9.787/99 } \\
\hline Nomenclatura do medicamento por princípio ativo & 250 & 14,6 \\
\hline \multicolumn{3}{|l|}{ Portaria $n^{0} 344 / 98$} \\
\hline Conformidade entre tipo prescrição e categorização do medicamento & 0 & 0 \\
\hline Conformidade entre tipo de prescrição e quantidade de medicamento & 1 & 0,01 \\
\hline Conformidade entre tipo de prescrição e quantidade de unidades posológicas & 2 & 0,02 \\
\hline Identificação da pessoa que recebeu o medicamento & 0 & 0 \\
\hline
\end{tabular}

FA: frequência absoluta; FR: frequência relativa

Figura 1. Distribuição das prescrições de medicamentos sujeitos a controle especial quanto à conformidade com a legislação, em receitas emitidas no período de julho de2018 a junho de 2019, arquivadas na Central de Abastecimento Farmacêutico (CAF) de Caicó, RN, Brasil.

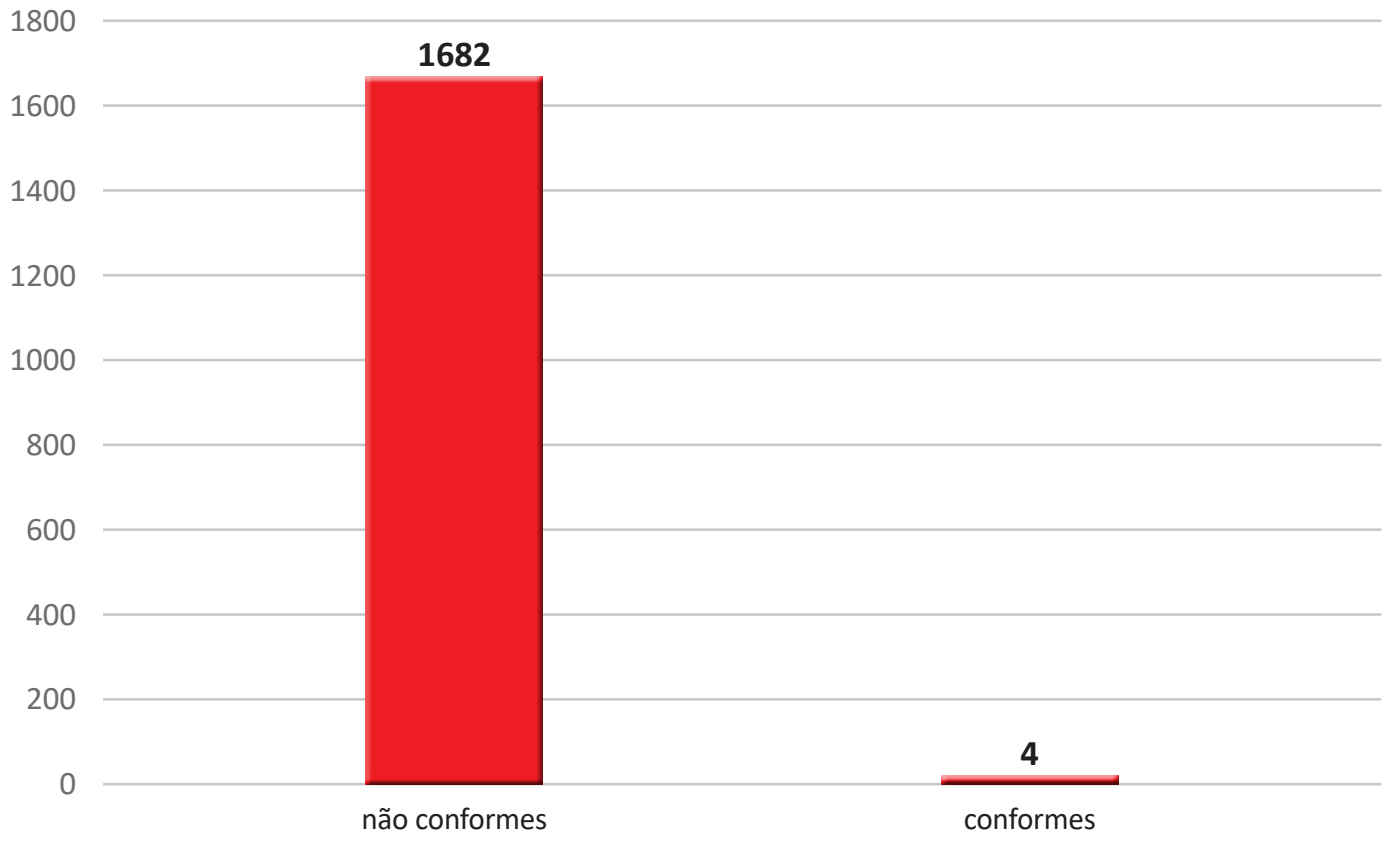


farmacêutico, principalmente, quanto a quantidade de medicamento e forma farmacêutica, respectivamente, a serem aviadas, o que contribui para inviabilização do processo de dispensação, consequentemente, para comprometimento da terapia farmacológica.

Por outro lado, esses dados superam os constatados em outros estudos, como o de avaliação de notificações de receita $B$ em Farmácia Básica de Pontal do Araguaia, MT, em que 15,5\% não continham informações posológicas (19), o de avaliação de prescrições em drogaria de São José do Rio Preto, SP, com $40 \%$ de prescrições com informações posológicas incompletas ou omissas (20) e o de avaliação de receituário de controle especial em drogaria de Recife, PE, em que 58\% não continham duração do tratamento (21).

Em um estudo de avaliação comparativa da compreensão de usuários da atenção primária sobre seu tratamento farmacológico no modelo de atendimento básico tradicional e na estratégia de saúde da família (ESF) foi constatado que usuários da ESF compreendiam melhor todos os itens da prescrição, exceto modo de administração, que foi considerado equivalente nos dois grupos supracitados (22).

Outro estudo, envolvendo a compreensão de prescrições de medicamentos por pacientes, comparando autorrelato do usuário com aferição do pesquisador, mostrou que $85,5 \%$ relataram ter compreendido todos os dados das prescrições, enquanto uma aferição do pesquisador indicou que $33,3 \%$ compreenderam todos os dados (nome do medicamento, número de doses diárias e horário de administração (23).

Todos os achados anteriormente mencionados subsidiam a hipótese de que a posologia constitui item de caráter de compreensão mais complexo, portanto, sendo essenciais a orientação ao usuário e a informação constante na prescrição a respeito deste item.

Por outro lado, a não conformidade quanto à duração do tratamento, em especial, pode configurar contexto de assistência à saúde marcado por renovação de prescrições sem avaliação clínica prévia, tendendo a ser mais presente quando há rotatividade/falta de médicos, carência de apoio matricial, deficiência na disponibilidade de recursos terapêuticos e alargamento de intervalos entre as reavaliações (24-28).

Considerando a nomenclatura do medicamento por princípio ativo, $14,6 \%$ das prescrições avaliadas estavam não conformes, o que corrobora revisões sistemáticas de avaliação de indicadores de prescrição de medicamentos na atenção primária, nas quais foi constatado que todos os estudos analisados continham medicamentos não prescritos pelo nome genérico $(29,30)$. Este fato contraria a meta da OMS de $100 \%$ dos medicamentos prescritos pelo nome genérico (6), a fim de facilitar educação, informação e acesso ao medicamento e promover seu uso racional (31), bem como o preconizado pela Lei $n^{\circ}$ 9.787/99, que prescrições médicas e odontológicas, no âmbito do Sistema Único de Saúde (SUS), devem adotar, obrigatoriamente, Denominação Comum Brasileira (DCB), no caso, o princípio ativo do medicamento (12).

Ao todo, foram prescritos 1.734 medicamentos, distribuídos em 9 classes farmacológicas, havendo predomínio de ansiolíticos benzodiazepínicos (30,97\%) (Figura 2). Todos os medicamentos presentes nas prescrições avaliadas constavam na REMUME 2014 (17), sendo essa a versão vigente no referido município, durante o estudo. $\mathrm{O}$ fato de Caicó não dispor de Comissão de Farmácia e Terapêutica tem contribuído, em parte, para o fato de não se dispor de versão mais atual da REMUME.

Considerando o contexto brasileiro, por volta de $2 \%$ da população adulta são usuários crônicos de benzodiazepínicos (32), prevalecendo uso por mulheres, com tendência a aumento de uso relacionado ao envelhecimento, podendo este estar relacionado ao aumento dos casos de insônia nesta faixa da vida (33).

Além disso, estudo de investigação de distribuição e frequência de consumo de benzodiazepínicos nas capitais brasileiras constatou que o consumo de ansiolíticos de meia vida curta vem crescendo ao longo dos anos, principalmente, nas capitais de maior densidade demográfica e concentração de médicos, o que se deve à crescente medicalização da sociedade moderna, refletida na formação dos médicos (34).

Diante dessa conjuntura, investigação sobre intervenções de descontinuação de uso de benzo- 
Figura 2. Distribuição dos medicamentos prescritos quanto à classe farmacológica, em receitas emitidas no período de julho de2018 a junho de 2019, arquivadas na Central de Abastecimento Farmacêutico (CAF) de Caicó, RN, Brasil.

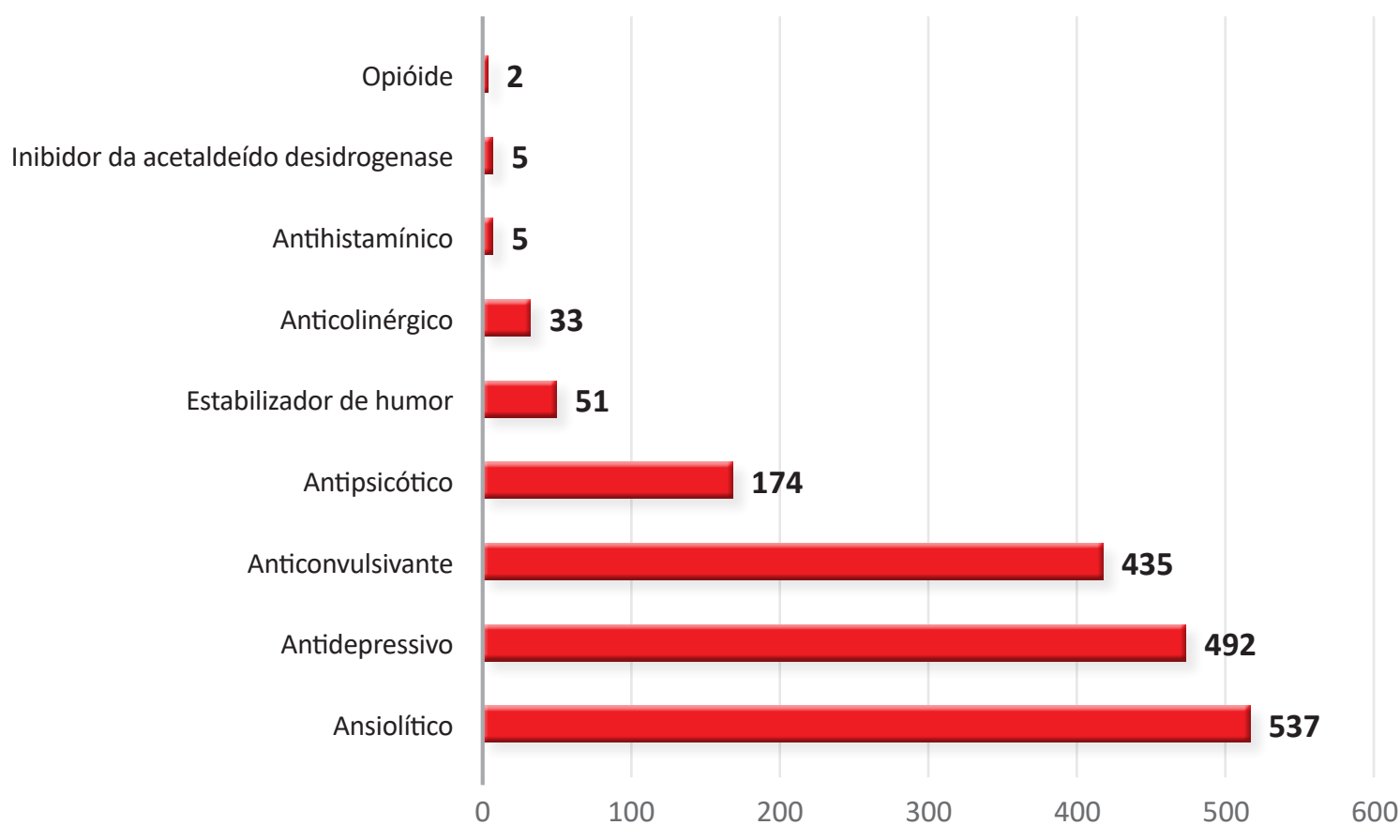

diazepínicos constatou que seu uso prolongado resulta de fatores como perfil de paciente e profissional, problemas pessoais e doenças crônicas e que estratégias para descontinuação incluem consultas domiciliares, terapia cognitivo-comportamental, prescrição de medicamentos que prolongam liberação de melatonina e aconselhamento via cartas referenciadas pelo médico. Descontinuar seu uso, entretanto, continua a ser um desafio, principalmente, para médicos da atenção primária (35).

\section{CONCLUSÃO}

Diante destes resultados, depreende-se a necessidade de reorientação da formação profissional médica, através de inserção e/ou maior ênfase a estratégias para elaboração de prescrições em observância a preceitos técnico-legais e usuário-cen- trados, a fim de contribuir para redução de risco de erros de medicação, consequentemente, melhoria da segurança do paciente.

Além disso, destes resultados emerge uma reflexão acerca da necessidade de reorientação da formação profissional no contexto das profissões de saúde, através de educação e trabalho interprofissionais, o que poderia contribuir, em parte, para melhoria da assistência à saúde na área de saúde mental, através, por exemplo, do processo de apoio matricial, o que contribuiria para melhoria do acesso e da efetividade quanto à assistência à saúde.

Desta forma, a assistência usuário-centrada, além de melhoria da segurança do paciente, do acesso e da efetividade, tende a contribuir, em contexto mais amplo, para melhoria da qualidade dos serviços de saúde. 
1. Fuchs FD, Wanmacher L. (Org.). Farmacologia clínica: fundamentos da terapêutica racional. 4. ed. Rio de Janeiro: Guanabara Koogan. 2010.

2. Santi LQ. Prescrição: o que levar em conta? In: Carvalho FD; Wannmacher L (ed.). Uso Racional de Medicamentos: fundamentação em condutas terapêuticas e nos macroprocessos da Assistência Farmacêutica. 1(14):1-12. 2016

3. WHO. World Health Organization. Medication Without Harm: WHO's Third Global Patient Safety Challenge. Genebra: OMS; 2017.

4. Rosa MB. Prevenção de erros de medicação: muito a ser feito no Brasil! Rev Bras Farm Hosp Serv Saude. 2019;10(3):1-2. DOI: 10.30968/rbfhss.2019.103.0449.

5. Marchon SG, Mendes Junior WV. Segurança do paciente na atenção primária à saúde: revisão sistemática. Cad. Saúde Pública. 2014;30(9):1-21. DOI: 10.1590/0102311X00114113.

6. WHO. World Health Organization. How to investigate drug use in health facilities: selected drug use indicators. Genebra: WHO. 1993.

7. WHO. World Health Organization. Patient safety. Medication without harm: WHO's third global patient safety challenge. 2017.

8. BRASIL. Portaria $n^{\circ} 529$, de $1^{\circ}$ de abril de 2013. Institui o Programa Nacional de Segurança do Paciente (PNSP). Diário Oficial da União, n. 62, seção 1, p. 43, 2013.

9. BRASIL.. Protocolo de segurança na prescrição, uso e administração de medicamentos. Brasilia: Ministério da Saúde 2013.

10. BRASIL. Lei $n^{\circ}$. 5.991, de 17 de dezembro de 1973. Dispõe sobre o controle sanitário do comércio de drogas, medicamentos, insumos farmacêuticos e correlatos, e dá outras providências. Diário Oficial da União, nº 7, 19 de dezembro de 1973. Seção 1. p. 13049.

11. BRASIL. Portaria ${ }^{\circ} 344$, de 12 de maio de 1998. Aprova o Regulamento Técnico sobre substâncias e medicamentos sujeitos a controle especial. Diário Oficial da União, 31 dez. 1998.

12. BRASIL. Lei $\mathrm{n}^{\circ} 9.787$, de 10 de fevereiro de 1999. Altera a Lei $n^{\circ} 6.360$, de 23 de setembro de 1976, que dispõe sobre a vigilância sanitária, estabelece o medicamento genérico, dispõe sobre a utilização de nomes genéricos em produtos farmacêuticos e dá outras providências. Diário Oficial da União, nº 2, 11 de fevereiro de 1999. Seção 1. p. 1.

13. BRASIL. Resolução n ${ }^{\circ} 20$, de 5 de maio de 2011. Dispõe sobre o controle de medicamentos à base de substâncias classificadas como antimicrobianos, de uso sob prescrição, isoladas ou em associação. Agência Nacional de Vigilância Sanitária. Diário Oficial da União, nº 87, 9 de maio de 2011. Seção 1. p. 39-41.

14. WHO. World Health Organization. Mental Health Atlas 2017. Geneva: WHO. 2018.

15. BRASIL. RN - Perfil territorial. Disponível em: http://sit. mda.gov.br/download/caderno/caderno_territorial_076_ Serid $\%$ C3\%83\%C2\%B3\%20-\%20RN.pdf.

16. WHO. World Health Organization. Anatomical Therapeutic Chemical (ATC): structure and principles. Geneva: WHO. 2013.

17. CAICÓ. Relação Municipal de Medicamentos Essenciais (REMUME). Secretaria Municipal de Saúde. 2014.

18. Mota IVR, Nascimento LES, Cardoso RA, Lemos LB, Lemos GS. Medicamentos sob controle especial: uma análise dos erros de medicação e indicadores de prescrição. Rev Eletr Farm. 2016;8(1):45-54. DOI: 10.5216/ref.v13i1.36594.

19. Ferrari CKB, Brito LF, Oliveira CC, Moraes EV, Toledo OR, David FL. Falhas na prescrição e dispensação de medicamentos psicotrópicos: um problema de saúde pública. Rev Ciênc Farm Básica Apl. 2013;34(1): 109-116.

20. Lima TAM, Gouveia MIS, Pereira LLV, Godoy MF. Erros de prescrições médicas em drogaria. Infarma Cien Farm. 2016;28(1):16-21. DOI: 10.14450/2318-9312. v28.e1.a2016.pp16-21.

21. Amaral AGS, Holanda FACL, Castro TM, Ferreira AS, Oliveira FFFG, Rolim Neto PJ, Silva RMF. Análise de prescrições irregulares em uma rede de farmácias do Recife. Rev. APS. 2019;22(2):251-263. DOI: 10.34019/ 1809-8363.2019.v22.16487.

22. Zanetti MOB, Marchetti JM, Andrade RCG. Compreensão do usuário da atenção primária à saúde sobre o tratamento farmacológico: uma análise comparativa. JAPHAC. 2020;(7):1-10.

23. Coradi CO, Cardoso JS, Groia RCZ, Silva KCL, Ceccato MGB, Lima MG. Compreensão da prescrição de medicamentos em uma unidade básica de saúde: autorrelato do usuário e aferição pelo pesquisador. Sci Med. 2016;26(4):1-6. DOI: 10.15448/1980-6108. 2016.4.24934.

24. Dimenstein M, Severo AK, Brito M, Pimenta AL, Medeiros V, Bezerra E. O apoio matricial em unidades de saúde da família: experimentando inovações em saúde mental. Saude Soc. 2009;18(1):63-74.

25. Campos RO, Gama CA, Ferrer AL, Santos DVD, Stefanello S, Trapé TL, Porto K. Saúde mental na atenção primária à saúde: estudo avaliativo em uma grande cidade brasileira. Cien Saude Colet. 2011;16(12): 4643-4652. DOI: 10.1590/S1413-81232011001300013. 
26. Frosi RV, Tesser CD. Práticas assistenciais em saúde mental na atenção primária à saúde: análise a partir de experiências desenvolvidas em Florianópolis, Brasil. Ciênc. Saúde Colet. 2015; 20(10):3151-3161. DOI: 10.1590/1413-812320152010.10292014.

27. Vasconcelos AMA, Silva DG. Concepção de cuidados em saúde mental na atenção primária à saúde. R. Interd. 2017;10(3):71-77.

28. Fegadolli C, Varela NMD, Carlini ELA. Uso e abuso de benzodiazepínicos na atenção primária à saúde: práticas profissionais no Brasil e em Cuba. Cad. Saúde Pública. 2019;35(6):1-11. DOI: 10.1590/0102-311X000 97718.

29. Souza PHR, Oliveira MAS. Indicadores de prescrição médica: uma revisão sistemática. 2015;27(1):5-13. DOI: 10.14450/2318-9312.v27.e1.a2015.pp5-13.

30. Silva AS, Maciel GA, Wanderley LSL, Wanderley AG. Indicadores do uso de medicamentos na atenção primária de saúde: uma revisão sistemática. Rev Panam Salud Publica. 2017;41:1-12.
31. OMS. Organização Mundial da Saúde. Guia para a boa prescrição médica. Porto Alegre: Artmed. 1998.

32. Firmino KF, Abreu MHNG, Perini E, Magalhães SMS. Fatores associados ao uso de benzodiazepínicos no serviço municipal de saúde da cidade de Coronel Fabriciano, Minas Gerais, Brasil. Cad Saúde Pública. 2011;27(6):1223-1232. DOI: 10.1590/S0102-311X2011 000600019 .

33. Fiorelli K, Assini FL. A prescrição de benzodiazepínicos no Brasil: uma análise da literatura. ABCS Health Sci. 2017;42(1):40-44. DOI: 10.7322/abcshs.v42i1.948.

34. Azevedo AJP, Araújo AA, Ferreira MAF. Consumo de ansiolíticos benzodiazepínicos: uma correlação entre dados do SNGPC e indicadores sociodemográficos nas capitais brasileiras. Cienc Saúde Colet. 2016;21(1): 83-90. DOI: 10.1590/1413-81232015211.15532014.

35. Carvalho MRF, Rodrigues ET, Golzio AMFO. Intervenções no uso prolongado de benzodiazepínicos: uma revisão. RSC. 2016; 5(2):55-64. DOI: 10.35572/rsc. v5i2.217. 